Methods Women older than 60 years with osteoporosis $(\mathrm{N}=$ 49) were enrolled in the study. All were able to walk indoor without technical support. Bone density was assessed using a DXA-machine.

All were hospitalised for five days in a hospital of rheumatology. They were casually put into two different treatment programs:

- Education $(\mathrm{n}=26)$

- Education in combination with physical training $(n=23)$

The education program was provided by a team of professional health workers and had a multifactorial approach to problems experienced by patient with osteoporosis. The physical training program was leaded by physiotherapists.

Health was assessed by SF-36 questionnaire at baseline, and then after 3 and 12 months.

Results A multivariate analysis for repeated measurements showed an improvement of bodily pain score of 7.4 (95\% CI 2.7-12.1) after 3 month and 9.8 (95\% CI 4.2-15.4) after 1 year compared with baseline. There were no significant differences between the groups. Physical activity and social function were also statistical improved at 3 month, but the improvement did not persist for the complete follow-up period.

Conclusion Participation a professional education program reduces the pain experience in women with osteoporosis, and the improvement appear to persist for at least one year. This result needs confirmation by further studies.

\section{SP0140 PSYCHOLOGICAL SUPPORT AND EDUCATION AS THE MAIN ASPECTS OF SYSTEMIC LUPUS ERYTHEMATOSUS "MY LIFE" PROGRAMME FOR PATIENTS}

K Nowicka-Sauer, M Koseda-Dragan, M Hebanowski. Connective Tissue Diseases Unit, Department of Family Medicine, Medical University of Gdansk, Gdansk, Poland

\subsection{6/annrheumdis-2001.1260}

Background The objective of our previous research was to investigate the main problems, needs and emotional state of patients with SLE. We found that the sensation of limited knowledge of the disease, mood disturbances, depression, anxiety, pain and fatigue are the most common problems of our patients. As we know, the psychological wellbeing and the attitude towards the disease are important factors influence individuals' lives. In connexion with it we tried to create the programme which would help our patients to understand their illness, to improve their "living with lupus" and to find the more effective and satisfying way of coping with SLE (if needed). In this work we are presenting the main goals of the SLE "MY LIFE" PROGRAMME including medical and possible psychological consequences of lupus and methods which we are going to use in our programme.

Objectives of the SLE “My Life" programme The most important aims are:

- to increase knowledge about the disease

- to reduce anxiety

- to improve self-care and self-management

- to "wake up" an activity and independence

- to increase psychological self-awareness

- to give support

- to activate family members and/or friends
Methods 1. "What is lupus?" - "Education meetings" for patients and patients' family members and/or friends with physician and psychologist

2. "What does lupus mean to me?" - oral sessions: learning self-observation skills referring to:

- pain

- fatigue

- symptoms of the disease and flare symptoms

- emotions/feelings/thoughts - what do the patients think of e.g. their fears/anger, relatives/relationship, life/future etc.

3. "What can I do?" - "active session": practising self-observation and self-care results - "My experiences, my success".

4. "Am I satisfied?" - the evaluation of the programme.

The patients' satisfaction and the effectivity of the programme are going to be evaluated.

\section{SP0144 RESIDENTIAL WORKSHOP FOR PARENTS OF ADOLESCENTS WITH JUVENILE IDIOPATHIC ARTHRITIS: A PRELIMINARY EVALUATION}

AP Turner, JH Barlow, CC Wright. Psychosocial Research Centre, Coventry University, Coventry, UK

\subsection{6/annrheumdis-2001.1261}

Background Caring for a child who has a chronic health condition can be a difficult and demanding task. Research has shown that some parents are at an increased risk for psychosocial problems.

Objectives The purpose of this study was to evaluate the effectiveness of a pilot Workshop designed specifically for parents of children with JIA.

Method 26 parents attended the two-day residential Workshop. The Workshop aimed to provide opportunities for parents to meet and discuss issues pertinent to caring for a disabled child. The Parents of Children With Disabilities Inventory (PCDI) was used to assess perceived disability-related stress. The PCDI comprises 4 subscales: Medical and Legal Concerns, Concerns for the Child, Concerns for the Family, Concerns for the Self. Higher scores indicate greater frequency and worry. Results were compared against a Comparison Group (CG) of 28 parents who expressed an interest in but did not attend the Workshop.

Results Median decreases in scores were significantly greater for the Workshop Group (WG) compared with the CG for Concerns for the Self (frequency) and Total frequency scores, $(\mathrm{p}=$ $0.020,013$, respectively). For the WG there were significant group decreases over time for Concerns for the Child (worried), Concerns for the Self (frequency), Total frequency score and Total worried score, $(\mathrm{p}=0.023,0.044,0.031,0.009$, respectively).

Conclusion The finding of statistically significant improvements across a range of parental concerns is encouraging. Workshops for parents of children with JIA appear worthy of continued development and evaluation.

\section{SP0153 COOPERATING THE BALTIC STATES (LATVIA)}

KA Carselind. Vice Chairman of the Board, The Swedish Rheumatism Association, Osterskar, Sweden

10.1136/annrheumdis-2001.1262 